\title{
ON THE COMPLEXITY OF RADIO RESOURCES ALLOCATION IN WCDMA SYSTEMS
}

\author{
Emmanuelle Vivier ${ }^{1}$, Michel Terré ${ }^{2}$, Bernard Fino ${ }^{2}$ \\ ${ }^{2}$ ISEP, 21 rue d'Assas, 75006 Paris $\quad{ }^{2}$ CNAM, 292 rue Saint Martin, 75003 Paris
}

\begin{abstract}
Recent attention to resource allocation algorithms for multiservice CDMA networks has focused on algorithms optimizing the aggregate throughputs (sum of all individual throughputs) on the uplink and on the downlink. Unfortunately, for a given set of real time (RT) and non real-time (NRT) communications services, such optimal algorithms involve non-integer spreading factors that do not belong to a finite set of spreading length as used in $3 \mathrm{G}$ systems. In this paper, we propose four algorithms for power and spreading allocation to RT and NRT services implementable in a real CDMA network like UMTS in the Frequency Division Duplexing (FDD) mode. On the downlink, two algorithms are presented; the first one maximises the aggregate downlink NRT throughput whereas the second one maximises the number of simultaneously transmitted NRT services. On the uplink, an algorithm that maximises the aggregate uplink NRT throughput and a suboptimal one, more easy to implement, are presented. Thanks to power control, both algorithms allow more simultaneous transmitting terminals than the optimal one. In both directions, the resulting aggregate NRT throughputs are very close to the ones obtained by the optimal algorithms. The small difference is the price paid for obtaining truly assignable spreading factors.
\end{abstract}

\section{INTRODUCTION AND SYSTEM MODEL}

Wireless multimedia services in $3 \mathrm{G}$ networks are characterized by different quality of service requirements. The radio resource management problem in CDMA networks ${ }^{7}$ is closely connected to the necessity of power control to maximize the number of terminals supported by such systems, hence for increasing cellular capacity. Unlike TDMA systems, radio resources are not countable but could be seen as different rates allocated to active services. The maximum individual rate for each transmitter is theoretically bounded by the use of one OVSF (Orthogonal Variable Spreading Factor) tree for spreading data. More, the use of such spreading sequences makes data rates belong to a finite set of values. In this context, two main QoS classes, related to (RT) and (NRT) services are considered in a given cell. Resources 
allocation process aims at detemining a set of OVSF codes that ensures to each communication a correct transmission. Therefore, the process has to be compliant with some constraints:

1) the allocation process occurs very periodically, every $10 \mathrm{~ms}$ in the UMTS FDD Terrestrial Radio Accesss Network (UTRAN FDD) ${ }^{1 !}$.

2) priority is given to RT communications ${ }^{1,2}$.

3) uplink NRT services use the leftover capacity ${ }^{3}$ and downlink NRT services use the remaining transmission power of the base station.

4) each signal has to maintain a minimum target signal to noise-plusinterference ratio in order to be correctly demodulated. This threshold is slightly higher than the minimum operating point in order to take into consideration the random variations of the interference level ${ }^{3,5}$.

The following notations are used:

\begin{tabular}{|l|l|}
\hline$I_{\text {inter }}$ & Intercell interferences \\
\hline$I_{t h}$ & Thermal noise in a $3,84 \mathrm{MHz}-$ large band $\left(I_{t h}=-108 \mathrm{dBm}\right)$ \\
\hline$I$ & $I=I_{\text {inter }}+I_{t h}$ \\
\hline$P_{\max }$ & Maximum transmission power of the base station \\
\hline$p_{\max }$ & Maximum transmission power of the RT and NRT terminals \\
\hline$M$ & Number of NRT services uniformly distributed in the cell \\
\hline$Q$ & Number of RT services uniformly distributed in the cell \\
\hline$p_{i,} p^{\prime}{ }_{i}$ & $\begin{array}{l}\text { Transmission power allocated } \\
\text { for the transmission of the } i^{\text {th }} \text { NRT, RT service (downlink) } \\
\text { to the terminal transmitting the } i^{\text {th }} \text { NRT, RT service (uplink) }\end{array}$ \\
\hline$g_{i,} g_{i}{ }_{i}$ & $\begin{array}{l}\text { Channel gain between the terminal transmitting the } i^{\text {th }} \text { NRT, } \\
\text { RT service and the base station, and vice-versa }\end{array}$ \\
\hline$N_{i}$ & Spreading factor for the $i^{\text {th }}$ NRT service \\
\hline$N_{R T}$ & Spreading factor for RT communications \\
\hline$\alpha$ & $\begin{array}{l}\text { normalized cross-correlation between the spreading codes at } \\
\text { the receiver level } \\
0 \leq \alpha \leq 1\end{array}$ \\
\hline$\Gamma_{N R T}$ & $\begin{array}{l}\text { Minimum signal to noise-plus-interferences ratio to be } \\
\text { reached for NRT services }\end{array}$ \\
\hline$\Gamma_{N R T}(i)$ & signal to noise-plus-interferences ratio for the $i^{\text {th }}$ NRT service \\
\hline$\Gamma_{R T}(i)$ & signal to noise-plus-interferences ratio for the $i^{\text {th }}$ RT service \\
\hline$\Gamma_{R T}$ & $\begin{array}{l}\text { Minimum signal to noise-plus-interferences ratio to be } \\
\text { reached for RT services }\end{array}$ \\
\hline
\end{tabular}

It must be noticed that $\Gamma_{N R T}$ and $\Gamma_{R T}$ are the same for all the concerned terminals and that, for simplicity reasons, $N_{R T}$ is the same for all RT communications, as well as $\alpha$ for all cross-correlations.

NRT terminals are sorted in decreasing order of $g_{i}$ : the transmission channel quality is a decreasing function of $i, 1 \leq i \leq M$.

The algorithms optimizing the aggregate throughputs on the downlink and on the uplink ${ }^{3}$ involve non-integer spreading factors that do not belong to a 
finite set of spreading length as used in $3 G$ systems. They are described respectively in section 2 and 3. In addition, in the context of an UMTS FDD network, section 2 describes the proposed algorithms for the downlink and is concluded by the comparison of the performances of those algorithms. In the same context, section 3 presents two adaptations of the theoretical optimal algorithm on the uplink: an optimal one and a suboptimal one, and compares both performances. Finally, section 4 presents our conclusions.

\section{DOWNLINK}

\subsection{Algorithms}

On the downlink, $\Gamma_{N R T}$ and $\Gamma_{R T}$ can be expressed as follows:

$$
\Gamma_{N R T}(i)=N_{i} p_{i} g_{i}\left[I+\alpha\left(\sum_{k=1}^{Q} p_{k}^{\prime}+\sum_{\substack{k=1 \\ k \neq i}}^{M} p_{k}\right) g_{i}\right]^{-1} \geq \Gamma_{N R T}
$$

and

$$
\Gamma_{R T}(i)=N_{R T}{p^{\prime}}_{i} g_{i}^{\prime}\left[I+\left.\alpha\left(\sum_{k=1}^{M} p_{k}+\sum_{\substack{k=1 \\ k \neq i}}^{Q} p_{k}^{\prime}\right) g_{i}^{\prime}\right|_{\rfloor} ^{-1} \geq \Gamma_{R T}\right.
$$

First, the amount of the transmission power dedicated to RT communications must be determined. For this purpose, interferences generated for NRT services must be estimated. In the worst case,

$$
P_{\max }=\sum_{k=1}^{M} p_{k}+\sum_{k=1}^{Q} p_{k}^{\prime}
$$

Transmission power allocated for the transmission of the $i^{\text {th }}$ RT service is therefore straightforward to reach $\Gamma_{R T}(i)=\Gamma_{R T}$ :

$$
p_{i}^{\prime}=\Gamma_{R T}\left(I+\alpha P_{\max } g_{i}^{\prime}\right)\left[\left(N_{R T}+\alpha \Gamma_{R T}\right) g_{i}^{\prime}\right]^{-1}
$$

Then, the remaining transmission power of the base station can be allocated to NRT services. Several allocation policies are conceivable. The aggregate downlink rate is: $\Omega_{N R T}^{\downarrow}=\sum_{i=1}^{M} 7,68 / N_{i}$, in Mbits/s. Actually, a constant chip rate (including the radio supervision) of 5120 chips per $10 / 15 \mathrm{~ms}$ is performed. 
The optimal allocation, maximising $\Omega_{N R T}^{\downarrow}$ consists in allocating all the remaining transmission power for the NRT service benefiting from the highest channel quality: $p_{1}=P_{\max }-\sum_{i=1}^{Q} p_{i}^{\prime}=P_{\max }-P_{R T}$

It leads to:

$$
N_{1}=\Gamma_{N R T}\left(I+\alpha g_{1} P_{R T}\right)\left[\left(P_{\max }-P_{R T}\right) g_{1}\right]^{-1}
$$

and $\Omega_{N R T}^{\downarrow *}=7,68 / N_{1}$.

Unfortunately, $N_{1}$ has no reason to be in the set of values $\mathrm{SF}_{\downarrow}=\{4,8,16,32$, $64,128,256,512\}$ that have been normalized for UTRAN downlink ${ }^{10}$. Consequently, $\Omega_{N R T}^{\downarrow *}$ is a theorical upper bound for $\Omega_{N R T}^{\downarrow}$.

In the following, we propose two algorithms. Under the constraint of spreading factors belonging to $\mathrm{SF}_{\downarrow}$, and always considering NRT services in the decreasing order of their channel gain,

1) the first algorithm, named Downlink Discrete Spreading Factor Up ('DownlinkDSF-U'), maximises the aggregate downlink rate by allocating to the considered NRT service the lowest spreading factors that leads to a 'feasible' solution. Once a speading factor is allocated, it is not modified any more even when considering a following NRT service.

2) the second algorithm, named Downlink Discrete Spreading Factor Down ('DownlinkDSF-D'), maximises the number of simultaneous transmitted NRT services by allocating the highest spreading factor of $\mathrm{SF}_{\downarrow}$ (i.e 512) to NRT services as long as it leads to a 'feasible' solution. Then, the number of simultaneous transmitted NRT services being fixed, it rises, while it is feasible, the individual rate of each service one step by one step.

The 'feasibility' of a solution is now defined: when $N_{1}, N_{2}, \ldots N_{\mathrm{m}}$ are known, the transmission power allocated for the transmission of the $i^{\text {th }}$ NRT service, $1 \leq i \leq m \leq M$ is determined as follows:

From (1) we obtain, to reach $\Gamma_{N R T}$ :

$$
p_{i}=\Gamma_{N R T}\left(I+\alpha g_{i} P_{T}\right)\left[g_{i}\left(N_{i}+\alpha \Gamma_{N R T}\right)\right]^{-1}
$$

where $P_{T}=\sum_{k=1}^{Q} p^{\prime}{ }_{k}+\sum_{k=1}^{m} p_{k}=P_{R T}+\sum_{k=1}^{m} p_{k} \leq P_{\max }$

Therefore:

$P_{T}-P_{R T}=\Gamma_{N R T}\left(I \sum_{i=1}^{m}\left[g_{i}\left(N_{i}+\alpha \Gamma_{N R T}\right)\right]^{-1}+\alpha P_{T} \sum_{i=1}^{m}\left[N_{i}+\alpha \Gamma_{N R T}\right]^{-1}\right)$ 
and

$$
P_{T}=\frac{P_{R T}+\Gamma_{N R T} I \sum_{i=1}^{m}\left[g_{i}\left(N_{i}+\alpha \Gamma_{N R T}\right)\right]^{-1}}{1-\alpha \Gamma_{N R T} \sum_{i=1}^{m}\left[N_{i}+\alpha \Gamma_{N R T}\right]^{-1}}
$$

If $\alpha \Gamma_{N R T} \sum_{i=1}^{m}\left[N_{i}+\alpha \Gamma_{N R T}\right]^{-1}<1$ and $P_{T} \leq P_{\max }$, the solution is 'feasible' and $p_{i}$ is obtained with (6) and (7).

\subsection{Performances}

RT and NRT terminals are uniformly distributed in the cell for the distance from the base station from $325 \mathrm{~m}$ to $1.2 \mathrm{~km}$. In order to determine the channel gains, we chose Okumura-Hata propagation model in an urban area with $f=2 \mathrm{GHz}, h_{\text {Base station }}=40 \mathrm{~m}$ and $h_{\text {terminal }}=1.5 \mathrm{~m}^{6}$. Let $P_{\max }=10 \mathrm{~W}$ and $I_{\text {inter }}=-63 \mathrm{dBm}$ (equivalent to 6 base stations situated $2 \mathrm{~km}$ far away from the considered base statio and transmitting at $\left.P_{\max }\right) . \Gamma_{R T}$ and $\Gamma_{N R T}$ are set to $7,4 \mathrm{~dB}$. $Q$ is set to 50 and $M$ varies from 1 to 500 . Finally, $N_{R T}=256$ and $\alpha=0,5$.

Figure 1 illustrates the variations of $\Omega_{N R T}^{\downarrow *}, \Omega_{N R T}^{\downarrow}$ obtained with 'DownlinkDSF-U' and $\Omega_{N R T}^{\downarrow}$ obtained with 'DownlinkDSF-D'. Figure 2 gives the number of simultaneously transmitted NRT services with 'DownlinkDSF-U' and 'DownlinkDSF-D' as a function of the total number of active downlink NRT services in the cell $(M)$. It is recalled that with the theoretical optimal algorithm, only one NRT service is served.

It appears that when 'DownlinkDSF-D' is applied, the number of simultaneous transmitted NRT service is exactly $M$ when $M$ is low (typically lower than 25). All NRT services being transmitted, $\Omega_{N R T}^{\downarrow}$ first increases and then fluctuates, depending on the random distribution of the terminals. Then, as the base station uses all its power to reach more and more terminals benefiting from worse and worse conditions of propagation, it can not transmit information to all NRT services and the individual rates remain minimum. Therefore, the aggregate throughput is nearly proportional to the number of simultaneously served NRT services and never exceeds $550 \mathrm{kbits} / \mathrm{s}$.

On the opposite, the 'DownlinkDSF-U' never simultaneously transmit information to more than 4 NRT services, whose spreading factor is at least 32. More, the probability of having terminals benefiting from higher conditions of propagation increases with $M$ increasing. Hence, $\Omega_{N R T}^{\downarrow}$ and 
$\Omega_{N R T}^{\downarrow *}$ are increasing functions. Finally, $\Omega_{N R T}^{\downarrow}$ varies from 340 to $640 \mathrm{kbits} / \mathrm{s}$, i.e. from $73 \%$ to $90 \%$ of $\Omega_{N R T}^{\downarrow *}$.

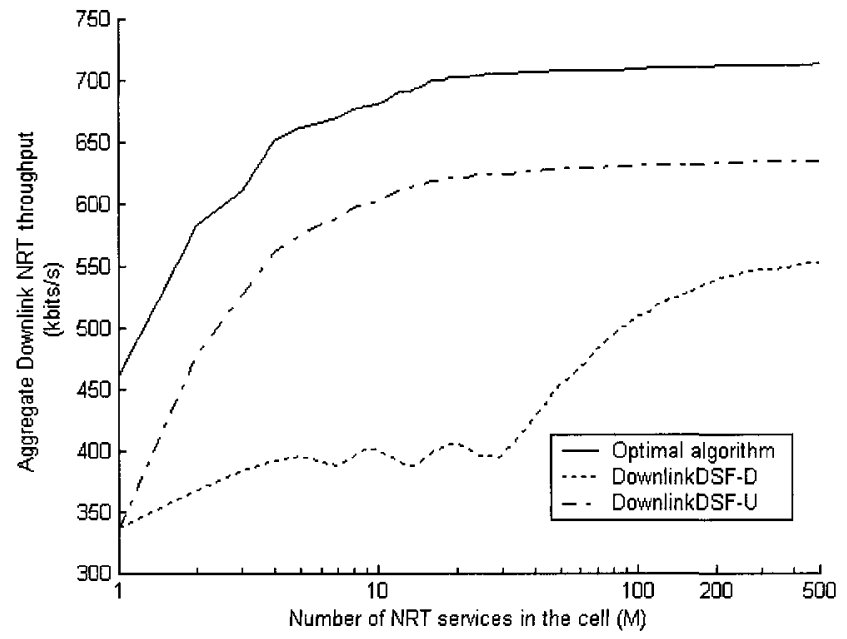

Figure 1: $\Omega_{N R T}^{\downarrow *}$ and $\Omega_{N R T}^{\downarrow}$ obtained with 'DownlinkDSF-U' and 'DownlinkDSF-D'

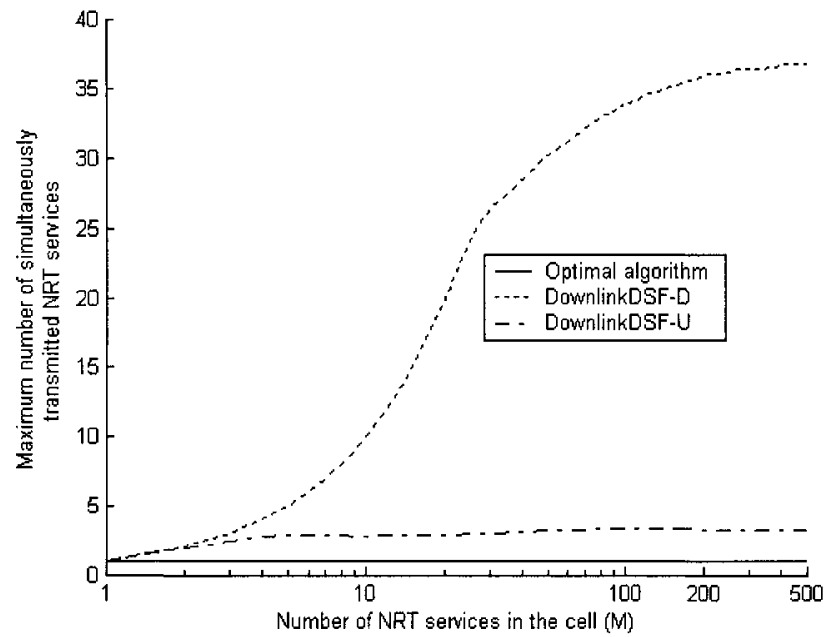

Figure 2: Number of simultaneously transmitted NRT services with 'DownlinkDSF-U' and 'DownlinkDSF-D', function of the total number of active NRT services in the cell $(M)$

The following section focuses on the UTRAN uplink. 


\section{UPLINK}

\subsection{Algorithms}

On the uplink, $\Gamma_{N R T}$ and $\Gamma_{R T}$ can be expressed as follows:

$$
\Gamma_{N R T}(i)=N_{i} p_{i} g_{i}\left[I+\alpha\left(\sum_{k=1}^{Q} p_{k}^{\prime} g^{\prime}{ }_{k}+\sum_{\substack{k=1 \\ k \neq i}}^{M} p_{k} g_{k}\right]^{-1} \geq \Gamma_{N R T}\right.
$$

and

$$
\Gamma_{R T}(i)=N_{R T}{p^{\prime}}_{i} g_{i}^{\prime}\left[I+\alpha\left(\sum_{k=1}^{M} p_{k} g_{k}+\sum_{\substack{k=1 \\ k \neq i}}^{Q} p_{k}^{\prime} g^{\prime}{ }_{k}\right]^{T^{-1}} \geq \Gamma_{R T}\right.
$$

As on the downlink, RT communications are still served first. Hence a maximum acceptable total power received by the base station from all NRT services $P_{R}^{N R T \max }$ is determined. This threshold represents the maximum value that ensures RT communications not to be blocked by NRT services. Therefore:

$$
\sum_{i=1}^{M} p_{i} g_{i} \leq P_{R}^{N R T \max }
$$

In the same way, $P_{R}^{R T}$ is the total power received by the base station from all RT services: $P_{R}^{R T}=\sum_{i=1}^{Q} p_{i}^{\prime} g_{i}^{\prime}$.

Consequently, in order to reach exactly $\Gamma_{R T}$, we obtain from (9):

$$
p_{i}^{\prime} g_{i}^{\prime}=\Gamma_{R T}\left(I+\alpha\left(P_{R}^{N R T \max }+P_{R}^{R T}\right)\right)\left[N_{R T}+\alpha \Gamma_{R T}\right]^{-1}
$$

and:

$$
P_{R}^{R T}=Q \Gamma_{R T}\left(I+\alpha\left(P_{R}^{N R T \max }+P_{R}^{R T}\right)\right)\left[N_{R T}+\alpha \Gamma_{R T}\right]^{-1}
$$

Therefore:

$$
Q=\left\lfloor\left. P_{R}^{R T}\left(N_{R T}+\alpha \Gamma_{R T}\right)\left[\Gamma_{R T}\left(I+\alpha\left(P_{R}^{N R T \max }+P_{R}^{R T}\right)\right)\right]^{-1}\right|_{\rfloor}\right.
$$

and from (11) $Q$ determines the real value of $P_{R}^{R T}$. Finally: 


$$
p_{i}^{\prime}=\Gamma_{R T}\left(I+\alpha\left(P_{R}^{N R T \max }+P_{R}^{R T}\right)\right)\left[\left(N_{R T}+\alpha \Gamma_{R T}\right) g_{i}^{\prime}\right]^{-1}
$$

Once transmission powers for RT communications are determined such that $0 \leq p_{i}{ }_{i} p_{\max }, p_{R}^{N R T \max }$ can be shared between NRT services.

Algorithms maximising the aggregate NRT uplink rate $\Omega_{N R T}^{\uparrow *}$ consider as on the downlink the NRT services in the decreasing order of their channel gain $^{3,4}$. While $\Omega_{N R T}^{\uparrow}(m)=\sum_{i=1}^{m} 3,84 / N_{i}$ (in Mbits/s, excluding the radio supervision) increases, they set $p_{i}$ to $p_{\max }$, except for the last NRT transmitting terminal of the cell because of (10). Once again, this process leads to non-integer numbers for spreading factors: as in the previous section, $\Omega_{N R T}^{\uparrow *}$ is the theoretical upper bound for $\Omega_{N R T}^{\uparrow}$. Actually, for the UTRAN, uplink spreading factors values must belong to $\mathrm{SF}_{\uparrow}=\{4,8,16,32$, $64,128,256\}^{10}$.

Therefore, in this paper, we propose an algorithm named Uplink Discrete Spreading Factor 'UplinkDSF' that ensures compatibility with UTRAN's spreading factors requirements. A perfect power control is considered. For a set of spreading factors $N_{1}, N_{2}, \ldots N_{\mathrm{m}}, 1 \leq m \leq M$, the corresponding transmission powers are determined as follows:

From (8) we obtain, to reach $\Gamma_{N R T}$ :

$$
p_{i} g_{i}=\left(\Gamma_{N R T}\left(I+\alpha P_{R}^{R T}+\alpha P_{R}^{N R T}\right)\right)\left[N_{i}+\alpha \Gamma_{N R T}\right]^{-1}
$$

where $P_{R}^{N R T}=\sum_{i=1}^{m} p_{i} g_{i}$. Therefore,

$$
P_{R}^{N R T}=\frac{\Gamma_{N R T}\left(I+\alpha P_{R}^{R T}\right) \sum_{i=1}^{m}\left[N_{i}+\alpha \Gamma_{N R T}\right]^{-1}}{1-\alpha \Gamma_{N R T} \sum_{i=1}^{m}\left[N_{i}+\alpha \Gamma_{N R T}\right]^{-1}}
$$

If $\alpha \Gamma_{N R T} \sum_{i=1}^{m}\left[N_{i}+\alpha \Gamma_{N R T}\right]^{-1}<1$ and $P_{R}^{N R T} \leq P_{R}^{N R T \max }, p_{i}$ is obtained with (13) and (14) .

Lastly, if $p_{i} \leq p_{\max }$, the solution is feasible. Otherwise, the spreading factor of the lowest path gain of the terminals that do not check $0 \leq p_{i} \leq p_{\max }$ is increased by one step. In this way, a new set of spreading factors is considered at the input of the algorithm. If the solution is feasible, 
a new NRT service is considered. Otherwise, the cell has reached its capacity.

\subsection{Performances}

The same uniform distribution of RT and NRT terminals as for the downlink is considered, as well as the determination of the channel gains. Let $I_{\text {inter }}=-$ $92.3 \mathrm{dBm}$ be the received power when $Q=50 \mathrm{RT}$ terminals transmit at $p_{\max }=23 \mathrm{dBm}^{9}$ with an attenuation of $132 \mathrm{~dB}$. Finally, $P_{R}^{N R T \max }=1,76 \mathrm{pW}$ is equivalent to $3 \times I, \Gamma_{R T}$ and $\Gamma_{\mathrm{NRT}}$ are set to $7,4 \mathrm{~dB}$ and $\alpha=0.5$. $M$ varies from 10 to 250 . Figure 3 gives the number of simultaneously transmitting uplink NRT services as a function of the number of active uplink services in the cell $(M)$. When $M$ is low, this number is higher with the optimal algorithm because the spreading factors values are not bounded by 256. $P_{R}^{N R T \max }$ is reached quite soon with the optimal algorithm because all transmitting terminals transmit at $p_{\max }$. Once $P_{R}^{N R T \max }$ is reached, the number of simultaneously transmitting uplink NRT services decreases. Actually, some terminals, well placed in the cell, can transmit at high rate and therefore generate quite a lot of received interference whereas others can not transmit without exceeding $p_{\max }$ with a spreading factor set to 256 (UplinkDSF) or without decreasing $\Omega_{N R T}^{\uparrow *}$. Finally, Figure 4 illustrates the variations of $\Omega_{N R T}^{\uparrow_{*}}$ and $\Omega_{N R T}^{\uparrow}: \Omega_{N R T}^{\uparrow}$ varies from $69 \%$ to $95 \%$ of $\Omega_{N R T}^{\uparrow *}$.

This UplinkDSF is easy to implement and gives very satisfactory results. However, it is not optimal. Consequently, the results of an optimal algorithm are also displayed. Of course, this 'Optimized UplinkDSF' algorithm ensures spreading factor values in $\mathrm{SF} \uparrow$. For each expected threshold of interferences lower than $P_{R}^{N R T \max }$ and for all NRT service (still in the decreasing order of their channel gains), it identifies individually all the spreading factors generating a level of interferences at the receiver side equal or lower than the expected threshold. Once the truly generated interferences are aggregated and checked lower than the expected threshold, it identifies the generated aggregated level of interferences equal or lower than $P_{R}^{N R T \max }$ that maximises the aggregate throughput. This approach is optimal and its complexity is closely connected to the number of analysed expected thresholds. For $25 \%$ of our results, a throughput about 3.84/256 Mbits/s higher than with the 'UplinkDSF' is obtained. It leads to a mean increase of 4 $\mathrm{kbits} / \mathrm{s}$ and corresponds to an $1 \%$ gain of throughput. 
The number of simultaneously transmitting uplink NRT services is approximately the same for both algorithms. Nevertheless, it can be noticed that 'UplinkDSF' allocates power of transmission to new NRT services as long as the resulting solution is feasible whereas 'Optimized UplinkDSF' stops at the maximum throughput. In conclusion, the 'UplinkDSF' algorithm is quasi-optimal in terms of throughput (it reaches $99 \%$ of the optimal one) and, when they are numerous in the cell, ensures more fairness among NRT services.

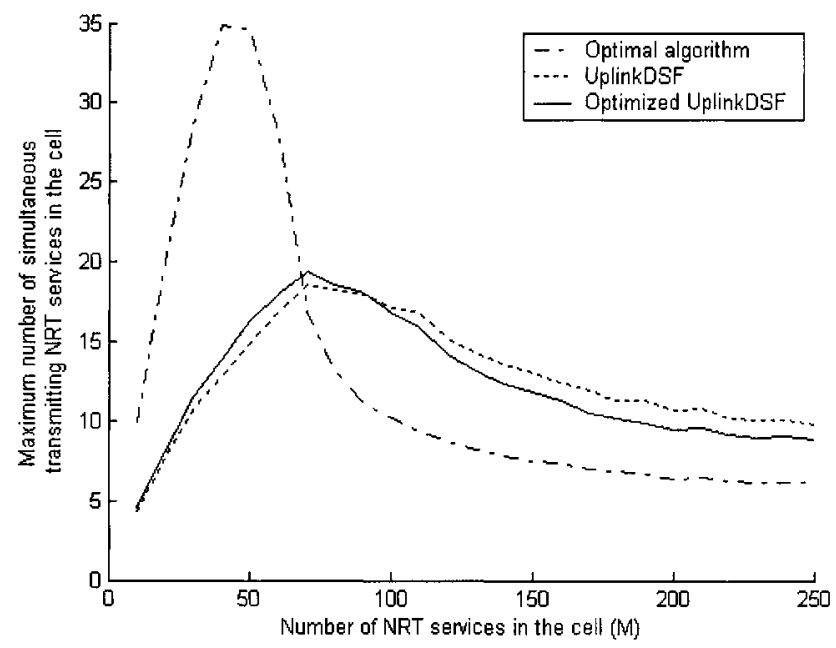

Figure 3: Number of simultaneously transmitting uplink NRT services, function of the number of active uplink services in the cell $(M)$ 


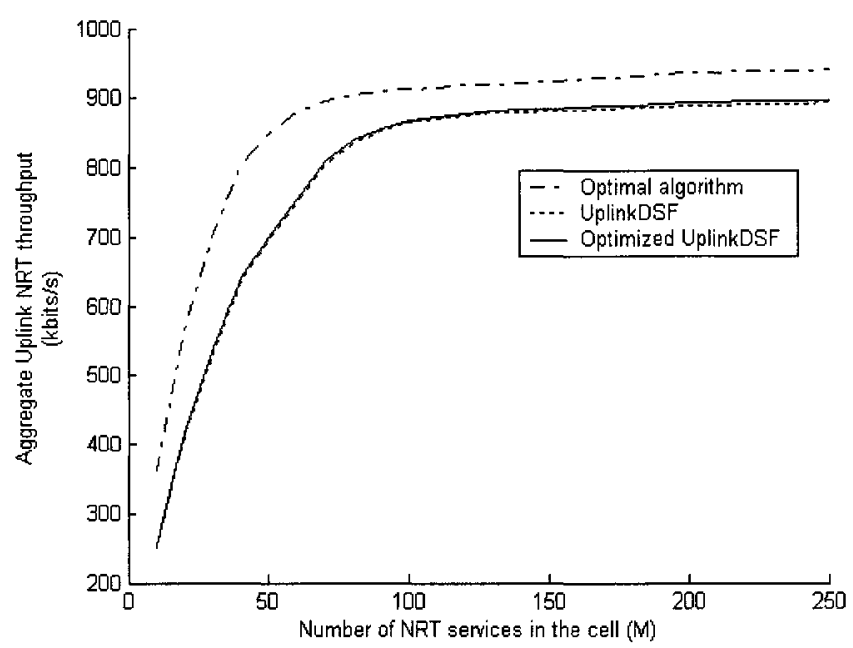

Figure 4: Variations of $\Omega_{N R T}^{\uparrow *}$ and $\Omega_{N R T}^{\uparrow}$

The radio resources allocation that maximises the aggregate NRT throughput of a CDMA network should gives on the downlink all the base station's power for the transmission of only one NRT service: the one benefiting from the best propagation conditions in the cell. On the uplink of such a system, while the aggregate throughput increases and the received interferences do not exceed a maximum threshold, it should allow NRT terminals that benefit from the best conditions of propagation to transmit at their peak power. But both algorithms lead to the determination of non integer values for spreading factors and therefore represent theoretical upper bounds.

In this paper, we proposed four algorithms for power and spreading allocation to RT and NRT services implementable in a real CDMA network like UMTS FDD. As for the determination of the theoretical upper bound of the aggregate rate, NRT users benefiting from the best conditions of propagation are the first served once RT communications are satisfied.

On the downlink, two algorithms were presented, allocating spreading factors in the set $\{4,8,16,3264,128,256,512\}$; 'DownlinkDSF-U' maximises the aggregate downlink NRT throughput whereas 'DownlinkDSF-D' maximises the number of simultaneously transmitted NRT services.

On the uplink, the proposed algorithm 'UplinkDSF' allocates spreading factors in the set $\{4,8,16,3264,128,256\}$ and gives a very interesting 
aggregate uplink NRT throughput. The optimal algorithm: 'Optimized UplinkDSF', whose complexity is more difficult to evaluate, leads to a hardly higher gain of the aggregate throughput. Thanks to power control, both algorithms allow more simultaneous uplink transmitting terminals than the theoretical optimal one.

With 'DownlinkDSF-U', 'UplinkDSF' and 'Optimized UplinkDSF', the resulting aggregate NRT throughputs are very close to the ones obtained by the optimal algorithms. The small difference is the price paid for obtaining truly assignable spreading factors.

\section{REFERENCES}

1. S. Choi and P. G. Shin, "An uplink CDMA system architecture with diverse QoS guarantees for heterogeneous traffic," IEEE Transactions on Networking, vol. 7, no. 5, oct. 1999.

2. O. Gürbüz, H. Owen, "Dynamic resource scheduling schemes for W-CDMA systems," IEEE Commun. Mag., Oct. 2000.

3. S. J. Oh, D. Zhang, and P. M. Wasserman, "Optimal Resource Allocation in Multiservice CDMA Networks," IEEE Trans. Wireless Commun., vol. 2, July 2003, pp. 811-821.

4. V. Rodriguez and D. J. Goodman, "Power and data rate assignment for maximal weighted throughput in 3G CDMA," in Proc. IEEE WCNC'03, pp. 525-531, vol.1.

5. T. Shu, Z. Niu, "Uplink capacity optimization by power allocation for multimedia CDMA networks with imperfect power control," IEEE J. Select Areas Commun., vol. 21, no. 10, pp. 1585-1594, Dec. 2003.

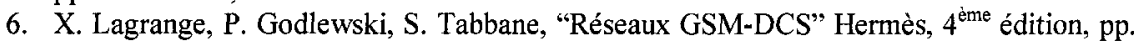
$141-142$.

7. J. Zander, "Radio resource management in future wireless networks: requirements and limitations," IEEE Commun. Mag., Aug. 1997

8. 3GPP TS 05.02, TSG GERAN, "Digital cellular telecommunications system (Phase 2+); Multiplexing and multiple access on the radio path".

9. 3GPP, Technical Specification 25.101 v3.16.0, UTRA (UE) FDD; Radio Transmission and Reception, Dec. 2003.

10. 3GPP, Technical Specification 25.211 v5.3.0, Physical channels and mapping of transport channels onto physical channels (FDD), December 2002.

11. 3GPP, Technical Specification 25.212 v5.3.0, Multiplexing and channel coding (FDD), December 2002. 